\title{
OPEN
}

Published online: 29 November 2019

\section{Author Correction: Comprehensive Analysis of Animal Models of Cardiovascular Disease using Multiscale X-Ray Phase Contrast Tomography}

\section{Hector Dejea, Patricia Garcia-Canadilla $\mathbb{D}$, Andrew C. Cook (D), Eduard Guasch (D), Monica Zamora, Fatima Crispi, Marco Stampanoni, Bart Bijnens (i) \& Anne Bonnin (iD)}

Correction to: Scientific Reports https://doi.org/10.1038/s41598-019-43407-z, published online 06 May 2019

The original version of this Article omitted an affiliation for Monica Zamora and Fatima Crispi.

The correct affiliations for Monica Zamora are listed below:

BCNatal, Hospital Clínic and Hospital Sant Joan de Déu, Barcelona, Spain

Institut d’Investigacions Biomèdiques August Pi i Sunyer (IDIBAPS), Barcelona, Spain

The correct affiliations for Fatima Crispi are listed below:

BCNatal, Hospital Clínic and Hospital Sant Joan de Déu, Barcelona, Spain

Centre for Biomedical Research on Rare Diseases (CIBER-ER), Hospital Clínic, Barcelona, Spain

Institut d’Investigacions Biomèdiques August Pi i Sunyer (IDIBAPS), Barcelona, Spain

Additionally, in the Supplementary information file an affiliation for Eduard Guasch was omitted.

The correct affiliations for Eduard Guasch are listed below:

Arrhythmia Unit, Department of Cardiology, Hospital Clínic de Barcelona, Barcelona, Spain

Institut d'Investigacions Biomèdiques August Pi i Sunyer (IDIBAPS), Barcelona, Spain

Centro de Investigación Biomédica en Red - Cardiovascular (CIBER-CV), Madrid, Spain

This has now been corrected in the HTML and PDF versions of this Article and in the Supplementary Information that accompanies this Article. 
(c) (i) Open Access This article is licensed under a Creative Commons Attribution 4.0 International License, which permits use, sharing, adaptation, distribution and reproduction in any medium or format, as long as you give appropriate credit to the original author(s) and the source, provide a link to the Creative Commons license, and indicate if changes were made. The images or other third party material in this article are included in the article's Creative Commons license, unless indicated otherwise in a credit line to the material. If material is not included in the article's Creative Commons license and your intended use is not permitted by statutory regulation or exceeds the permitted use, you will need to obtain permission directly from the copyright holder. To view a copy of this license, visit http://creativecommons.org/licenses/by/4.0/.

(C) The Author(s) 2019 